\section{Non-ophthalmologist screening for retinopathy of prematurity}

\author{
Richard A Saunders, Margaret L Donahue, Jerry E Berland, Eric L Roberts, \\ Billy Von Powers, Philip F Rust
}

ommended for routine clinical practice, this technique may nevertheless be of value to those situations where ophthalmological consultation is unavailable or difficult to obtain.

(Br f Ophthalmol 2000;84:130-134)

Retinopathy of prematurity (ROP) is a proliferative vascular disease characterised by abnormal blood vessel development and subsequent fibrosis in the peripheral retinas of prematurely born infants. ROP is a common disorder, occurring in up to $80 \%$ of infants born at 28 weeks' gestation or less, ${ }^{1}$ and can lead to serious long term vision loss and even blindness. ${ }^{23}$ Clinical studies have documented improved visual outcomes in infants with "threshold" ROP after treatment with either trans-scleral cryotherapy ${ }^{4}$ or transpupillary laser therapy. ${ }^{5-9}$ The American Academy of Pediatrics, the American Association for Pediatric Ophthalmology and Strabismus, and the American Academy of Ophthalmology recently released a joint statement recommending that initial screening examinations be performed in "at risk" infants between 4 and 6 weeks of chronological age or 31 weeks and 33 weeks of post-conceptional age. ${ }^{10}$ Other detection strategies have also been proposed. ${ }^{11}{ }^{12}$ These examinations are usually performed by a paediatric ophthalmologist, retinal specialist, or general ophthalmologist experienced in the diagnosis of infants with ROP.

Infants in many countries outside the United States, Western Europe, and Canada often fail to receive appropriate screening examination because of the unavailability of routine ophthalmological consultation. In a recent survey of schools for the blind in 23 "middle income" countries the proportion of severe visual impairment of blindness due to ROP ranged from $0 \%$ (most African countries) to $39 \%$ (Cuba). ${ }^{13}$ The authors recommend development of alternative screening programmes with guidelines appropriate to settings where routine ophthalmological consultation may assume a low priority because of the limited number of available ophthalmologists. Out of necessity, it follows that some of these examinations would have to be performed by non-ophthalmologist examiners. However,
Accepted for publication 28 September 1999 evaluating the posterior pole blood vessels of the retina. While not necessarily rec- 


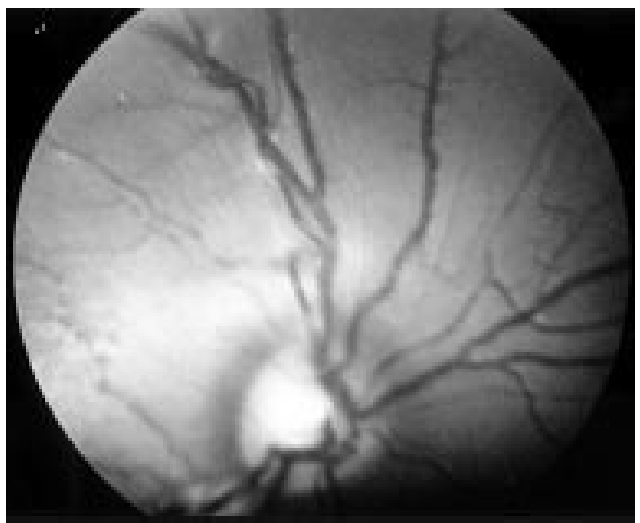

Figure 1 Funduscopic view of normal arterioles and venules in an infant of 35 weeks' gestational age (reprinted from Saunders et al ${ }^{14}$ with permission of Slack, Inc).

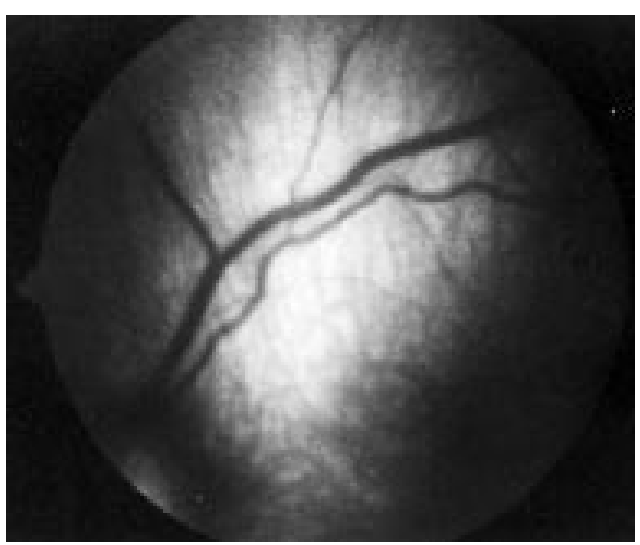

Figure 2 Funduscopic view of posterior pole blood vessels showing venous dilatation with normal arterioles (reprinted from Saunders et al ${ }^{14}$ with permission of Slack, Inc).

little is known about the potential effectiveness of alternative screening programmes.

A recent study in premature infants has shown a significant correlation between the appearance of the posterior pole blood vessels and the severity of peripheral retinal disease in ROP. ${ }^{14}$ Infants with normal appearing posterior pole vessels had mild or no ROP, lessening the need for concurrent indirect ophthalmoscopic examination of the peripheral retina. Examination of the posterior pole vessels can be accomplished using a standard hand held (direct) ophthalmoscope and, more importantly, potentially be performed by nonophthalmologists with reasonable accuracy after only minimal training. ${ }^{15}$ Our current study was undertaken as part of the training of a staff neonatologist (MLD) to evaluate the status of the posterior pole blood vessels in premature infant eyes. If deemed sufficiently reliable, preliminary screening by nonophthalmologists could be used to detect infants with evidence of progressive ROP who might then receive expedited referral to a specialist for evaluation and appropriate intervention.

\section{Subjects and methods}

Premature infants admitted to the intensive care nursery at the Medical University of South Carolina Children's Hospital between October 1993 and December 1994 (excluding
15 October to 7 December 1993) with birth weights less than $1600 \mathrm{~g}$, were enrolled in the study. Approval of the protocol was obtained by the university's institutional review board for human research. Routine screening examinations for ROP were performed before discharge or by 33 weeks post-conceptional age. Follow up examinations were performed as necessary, depending on clinical findings. Before each examination, the pupils were dilated with either Cyclomydril or sequential instillation of cyclopentolate $0.5 \%$ and phenylephrine $\mathrm{HCl} 2.5 \%$. Using a Cook-style paediatric eyelid speculum, ${ }^{16}$ funduscopic examination was performed $30-60$ minutes later at the bedside.

A non-ophthalmologist (MLD) examined both retinas of each infant using a halogen bulb direct ophthalmoscope and recorded whether the blood vessels were normal or abnormal, based on reference photographs (Figs 1-4). Other than explanation of the fundus photographs and viewing several examples of vascular abnormalities through the teaching mirror of the indirect ophthalmoscope, the nonophthalmologist examiner received no indepth training in ophthalmoscopy or the evalu-

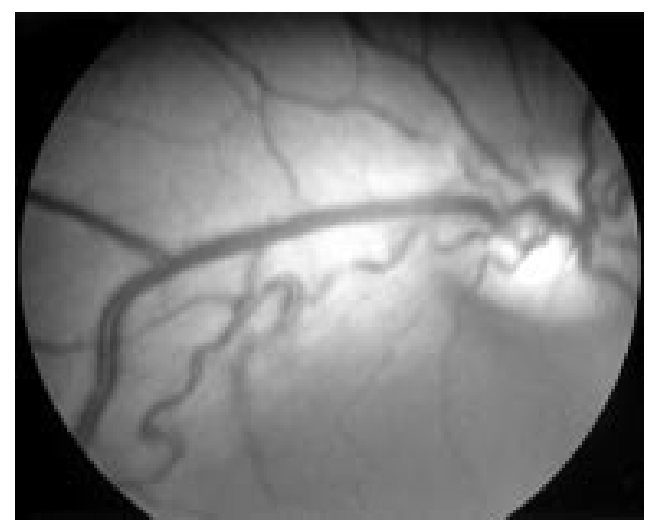

Figure 3 Funduscopic view of posterior pole vessels showing dilated venules, tortuous and minimally dilated arterioles, but not "plus disease" (reprinted from Saunders et al ${ }^{14}$ with permission of Slack, Inc).

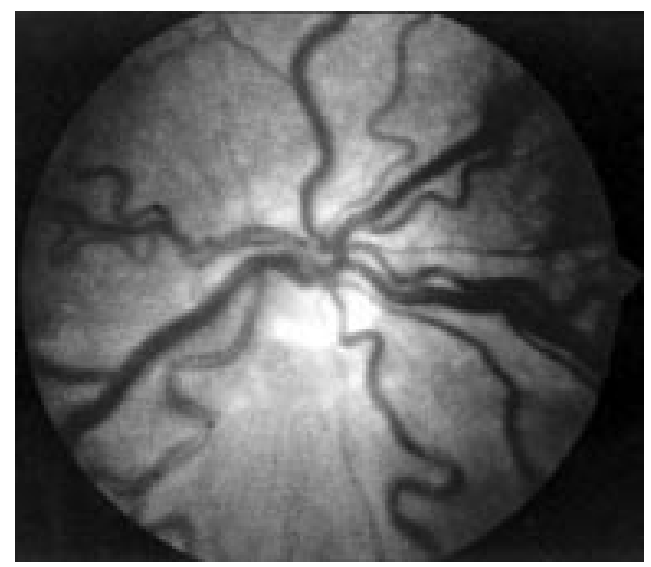

Figure 4 Funduscopic view of posterior pole vessels in moderate "plus disease." 17 This degree of dilatation and tortuosity is greater than the minimum standard for plus disease used in the Cryotherapy for Retinopathy of Prematurity Multicenter Clinical Trial ${ }^{2}$ (photograph courtesy of David B Schaffer, $M D$ and reprinted with permission of the Cryotherapy of Retinopathy of Prematurity Cooperative Group.) 
Table 1 Posterior pole vascular findings, ophthalmologist's versus non-ophthalmologist's examination

\begin{tabular}{lll}
\hline & \multicolumn{2}{c}{ Non-ophthalmologist's examination } \\
\cline { 2 - 3 } $\begin{array}{l}\text { Ophthalmologist's } \\
\text { examination }\end{array}$ & $\begin{array}{l}\text { Normal blood } \\
\text { vessels }\end{array}$ & $\begin{array}{l}\text { Abnormal blood } \\
\text { vessels }\end{array}$ \\
\hline $\begin{array}{l}\text { Normal posterior pole } \\
\text { Dilated venules }\end{array}$ & 31 & 16 \\
$\begin{array}{l}\text { Abnormal arterioles and } \\
\text { venules }\end{array}$ & 16 & 58 \\
Total & 0 & 21 \\
\hline
\end{tabular}

ation of posterior pole blood vessels before beginning this study. A vessel pair was considered abnormal if there was venous dilatation with or without accompanying dilatation or tortuosity of the retinal arterioles. Venules were considered dilated if they were greater than twice the calibre of normal appearing arterioles in the same eye, whereas the normal diameter ratio of venules to arterioles is approximately $3: 2$. Twelve eyes could not be evaluated using direct ophthalmoscopy because of vitreous haze, poorly dilated pupils, or inability to obtain adequate focus on the posterior pole structures.

After the non-ophthalmologist's findings had been recorded, a paediatric ophthalmologist (RAS) or paediatric ophthalmology fellow (JEB or ELR) examined both eyes using the indirect ophthalmoscope and graded the posterior pole blood vessels as 1 , normal, 2 , dilated venules, or 3 , dilated and tortuous arterioles and venules using the same photographic guidelines. Dilated and tortuous vessels did not necessarily imply that "plus disease" was present, ${ }^{2}$ but plus disease is included as a subset in this third group. Finally, an examination of the peripheral retina was performed using scleral depression and the findings recorded using the International Classification of ROP. ${ }^{17}$ The ophthalmologist examiner was not aware of the findings of the non-ophthalmologist examiner until each posterior pole examination was complete and the data recorded for both eyes. Infants with previously identified retinovascular abnormalities who were familiar to the non-ophthalmologist were excluded from the data analysis.

\section{Results}

Our results are summarised in Tables 1-4. A total of 142 infants were evaluated by both an ophthalmologist and non-ophthalmologist examiner. Twelve infants had incomplete examinations by the non-ophthalmologist because of inability to assess the posterior pole with the direct ophthalmoscope. These 12 infants are included in the "abnormal blood vessel" category in Tables 1 and 2. On peripheral retinal examination, eight had immature vessels without ROP, three had zone II, stage 2 ROP, and one had zone II, stage 3 ROP.

Table 1 shows the correlation between the non-ophthalmologist's interpretation of the posterior pole vessels and the ophthalmologist's findings for the same infants. Of the 142 infants examined by the non-ophthalmologist, $95(67 \%)$ were felt to have retinovascular abnormalities.
Table 2 Non-ophthalmologist's posterior pole vascular findings compared with severity of retinopathy of prematurity on peripheral retinal examination

\begin{tabular}{lcc}
\hline & $\begin{array}{l}\text { Normal blood } \\
\text { vessels }\end{array}$ & $\begin{array}{l}\text { Abnormal blood } \\
\text { vessels }\end{array}$ \\
\hline No ROP, mature & 12 & 11 \\
No ROP, immature & 30 & 36 \\
Zone III, stage 1 & 2 & 5 \\
Zone III, stage 2 & & 2 \\
Zone III, stage 3 & 2 & 12 \\
Zone II, stage 1 & 1 & $13^{\star}$ \\
Zone II, stage 2 & & $15 \dagger$ \\
Zone II, stage 3 & & \\
Zone I, stage 1 & & $1 \dagger$ \\
Zone I, stage 2 & & 95 \\
Zone I, stage 3 & 47 & Total
\end{tabular}

*One infant with zone II, stage 2 had "plus disease", consisten with prethreshold retinopathy of prematurity.

tAll of these infants were considered to have either prethreshold or threshold retinopathy of prematurity.

Of these, $16(17 \%)$ were judged by the ophthalmologist to have normal posterior pole blood vessels. By the non-ophthalmologist's examination, 47 infants had normal posterior pole blood vessels. Thirty one of these were also considered normal by the ophthalmologist, while 16 were found to have dilated venules. Importantly, all 21 infants with both abnormal arterioles and venules according to the ophthalmologist's examination were correctly identified as abnormal by the nonophthalmologist.

Table 2 compares the non-ophthalmologist's posterior pole vascular findings with the severity of ROP on peripheral retinal examination as determined by the ophthalmologist. None of the 17 children diagnosed with prethreshold or threshold ROP was thought to have normal posterior pole vessels by the nonophthalmologist. With one exception, all infants with ROP of zone II, stage 2 severity or worse were identified as having abnormal posterior pole vessels by the non-ophthalmologist.

Table 3 compares the ophthalmologists' posterior pole vascular findings with the severity of ROP on peripheral retinal examination. Dilated venules seemed to be a non-specific finding ${ }^{14} ; 16$ of 74 infants $(22 \%)$ with dilated venules had mature retinal vessels and 30 of 74 (41\%) had immature retinal vessels, but no evidence of ROP. The majority of infants $(77 \%)$ with prethreshold ROP had both

Table 3 Ophthalmologist's posterior pole vascular findings compared with severity of retinopathy of prematurity on peripheral retinal examination

\begin{tabular}{lccc}
\hline & $\begin{array}{l}\text { Normal } \\
\text { posterior pole }\end{array}$ & $\begin{array}{l}\text { Dilated } \\
\text { venules }\end{array}$ & $\begin{array}{l}\text { Abnormal } \\
\text { arterioles } \\
\text { and venules }\end{array}$ \\
\hline No ROP, mature & 5 & 16 & 2 \\
$\begin{array}{l}\text { No ROP, immature } \\
\text { Zone III, stage 1 }\end{array}$ & 36 & 30 & \\
$\begin{array}{l}\text { Zone III, stage 2 } \\
\text { Zone III, stage 3 }\end{array}$ & 1 & 6 & 1 \\
Zone II, stage 1 & 3 & 1 & \\
Zone II, stage 2 & 2 & 11 & $5^{\star}$ \\
$\begin{array}{l}\text { Zone II, stage 3 } \\
\text { Zone I, stage 1 }\end{array}$ & & $3 \dagger$ & $12 \dagger$ \\
Zone I, stage 2 & & & \\
Zone I, stage 3 & & & $1 \dagger$ \\
Total & 47 & 74 & 21 \\
\hline
\end{tabular}

*One infant with zone II, stage 2 had "plus disease", consistent with prethreshold retinopathy of prematurity.

tAll of these infants were considered to have either prethreshold or threshold retinopathy of prematurity. 
Table 4 Posterior pole vascular findings. Three dimensional frequency analysis of interreader interaction, non-ophthalmologist/retinopathy of prematurity interaction, and ophthalmologist/retinopathy of prematurity interaction

\begin{tabular}{llrlr}
\hline \multirow{2}{*}{$\begin{array}{c}\text { Non-ophthalmologist's } \\
\text { examination }\end{array}$} & Ophthalmologist's examination & & & \\
\cline { 2 - 5 } & Peripheral retinal findings & Normal & Abnormal & Total \\
\hline Normal blood vessels & Mature & 5 & 7 & 12 \\
& Immature or less than prethreshold & 26 & 9 & 35 \\
& Prethreshold or threshold & 0 & 0 & 0 \\
& Total & 31 & 16 & 47 \\
Abnormal blood & Mature & 0 & 11 & 11 \\
vessels & Immature or less than prethreshold & 16 & 51 & 67 \\
& Prethreshold or threshold & 0 & 17 & 17 \\
& Total & 16 & 79 & 95 \\
\hline
\end{tabular}

abnormal arterioles and venules, and the remaining $(23 \%)$ dilated venules but normal arterioles. By definition, all four infants with threshold ROP had abnormal arterioles and venules.

Table 4 is a three dimensional frequency analysis that shows three interactions: 1 , the non-ophthalmologist's evaluation of the posterior pole blood vessels correlated with the ophthalmologist's evaluation of the same vessels (interobserver interaction); 2 , the relation of the non-ophthalmologist's posterior pole findings with the peripheral retinal examination (non-ophthalmologist/ROP interaction); and 3 , the relation of the ophthalmologist's posterior pole diagnosis with the peripheral retinal findings using indirect ophthalmoscopy (ophthalmologist/ROP interaction). Using log linear analysis, each of these interactions is significant: interobserver interaction, $\chi^{2}=31.05$, $\mathrm{df}=1(\mathrm{p}<0.001)$; non-ophthalmologist/ROP interaction, $\chi^{2}=14.44, \mathrm{df}=2(\mathrm{p}<0.001)$; ophthalmologist/ROP interaction, $\chi^{2}=17.18$, $\mathrm{df}=2(\mathrm{p}<0.001)$. In this table, dilated venules and abnormal arterioles and venules are combined into the "abnormal" category for the ophthalmologists' findings since infants found to have any vascular abnormalities by the nonophthalmologist were considered abnormal. In each of the 17 prethreshold and threshold cases, both the non-ophthalmologist and the ophthalmologist considered the posterior pole vessels to be abnormal.

\section{Discussion}

Our results suggest that a non-ophthalmologist can be trained to screen premature infants for retinovascular abnormalities associated with severe ROP. While the standard of practice in the United States currently calls for ophthalmological screening for high risk premature infants, staffing considerations may make this impractical in other parts of the world. A screening protocol relying on nonophthalmologists could potentially be more comprehensive and cost effective by obviating the need for specialist consultation for every infant, yet allowing appropriate referral for the majority of high risk cases potentially requiring surgical intervention. In our experience, preventable blindness from ROP has almost always been associated with failure to perform appropriate screening or follow up examinations, not failure to diagnose correctly. Therefore, even in nursery environments where oph- thalmic consultation is more readily available, examination of posterior pole retinal blood vessels by non-ophthalmologists may still have diagnostic value.

Although a non-ophthalmologist screening protocol may be a useful clinical tool, there are several points that must be addressed. Firstly, consistent agreement on the diagnosis of dilated venules, even among ophthalmologists, was difficult to achieve. The comparison of the diameter of venules to arterioles was often borderline at a 2:1 ratio and might vary among vessel pairs within the same eye. In an emmetropic eye, image magnification with the direct ophthalmoscope is approximately five times greater than the indirect ophthalmoscope using a 20 dioptre condensing lens, and seven times greater than a 30 dioptre condensing lens. ${ }^{18} \mathrm{~A}$ certain amount of disagreement among examiners using different ophthalmoscopes would therefore be expected. Furthermore, during examination with the direct ophthalmoscope, often only one vessel pair can be adequately visualised. It is possible that the specific pair examined may or may not be abnormal, although vessel pairs that were not examined may have dilated venules or even arteriolar tortuosity. This problem can be overcome by examining more than one vessel pair in each fundus. Examiner persistence and perhaps prolonged or sequential examinations may sometimes be required.

While our high sensitivity for detecting posterior pole vascular abnormalities indicates that a non-ophthalmologist would not be likely to miss clinically important ROP on routine screening, specificity was poor. In this study, "abnormal" posterior pole blood vessels were identified in two thirds of infants undergoing screening examination by the nonophthalmologist examiner using a direct ophthalmoscope (Table 1). This would lead to many unnecessary referrals of low risk infants. Our arbitrary definition of dilated venules (greater than 2:1 ratio of the diameter of venules to arterioles) may therefore need to be revised to improve specificity in the correlating of posterior pole findings with important peripheral retinal disease. More precise grading of posterior pole vascular abnormalities, as we have proposed using the indirect ophthalmoscope, might also be possible. ${ }^{14}$

Secondly, dilatation of the pupils and use of an eyelid speculum is generally required regardless of whether a non-ophthalmologist or ophthalmologist performs the screening examination. Dilating eye drops need to be available in appropriate concentrations for premature infant to minimise potential medication side effect. The non-ophthalmologist will require appropriate training to place an eyelid speculum in the eye without scratching the cornea or damaging adnexal structures.

Thirdly, training of our non-ophthalmologist examiner using reference photographs and funduscopic examination through the teaching mirror of an indirect ophthalmoscope probably improved our results. Other nonophthalmologists interested in using this screening protocol would presumably require 
similar instruction to achieve an acceptable level of accuracy. Currently, there are no known programmes teaching these techniques to non-ophthalmologists. However, the experience needed to become competent in posterior pole vessel evaluation with the supervision of an ophthalmologist may be as few as $20-25$ examinations, assuming that these would include infants with varying degrees of abnormal posterior pole vascular findings.

It must also be stressed that the finding of normal posterior pole vessels at one point in time does not rule out the potential for developing blinding ROP later on. This screening protocol requires sequential examinations, documenting repeatedly normal posterior pole vessels or, alternatively, referral to an ophthalmologist for further evaluation. Previous studies on the natural progression of ROP have documented that the majority of infant retinas show mature vasculature by 38 weeks' post-conceptional age. ${ }^{1}$ It would therefore seem prudent that each high risk infant receive, where available, at one least one peripheral retinal examination by an ophthalmologist at approximately 38 weeks' postconceptional age to document retinal vascularisation into zone III. However, in countries where ophthalmological consultation is not routinely available and the incidence of cicatricial ROP is high, examinations by nonophthalmologists might represent the only screening a high risk infant receives before hospital discharge.

This study was supported in part by an unrestricted grant to the Storm Eye Institute from Research to Prevent Blindness, Inc, New York, USA.

1 Palmer EA, Flynn JT, Hardy RJ, et al. Incidence and early course of retinopathy of prematurity. Ophthalmology 1991;98:1628-40.
2 Cryotherapy for Retinopathy of Prematurity Cooperative Group. Multicenter trial of cryotherapy for retinopathy of prematurity: preliminary results. Arch Ophthalmol 1988; 106:471-9. (Identical publication in Pediatrics 1988;81: 697-706.)

3 Cryotherapy for Retinopathy of Prematurity Cooperative Group. The natural ocular outcome of premature birth and retinopathy. Arch Ophthalmol 1994;112:903-12.

4 Cryotherapy for Retinopathy of Prematurity Cooperative Group. Multicenter trial of cryotherapy for retinopathy of prematurity: Snellen visual acuity and structural outcome at 51/2 years after randomization. Arch Ophthalmol 1996; 114:417-24.

5 Landers MB, Toth CA, Semple C, et al. Treatment of retinopathy of prematurity with argon laser photocoagulation. Arch Ophthalmol 1992;110:44-7.

6 McNamara JA, Tasman W, Brown GC, et al. Laser photocoagulation for stage 3+ retinopathy of prematurity. Ophthalmology 1991;98:576-80.

7 Fleming TN, Runge PE, Charles ST. Diode laser photocoagulation for prethreshold posterior retinopathy of prematurity. Am 7 Ophthalmol 1992;114:589-92.

8 Capone A Jr, Diaz-Rohena R, Sternberg P Jr, et al. Diode-laser photocoagulation for zone I threshold retinopathy of prematurity. Am f Ophthalmol 1993;116:444-9.

9 Hunter G, Repka M. Diode laser photocoagulation for threshold retinopathy of prematurity: a randomized study. Ophthalmology 1993;100:238-44.

10 American Academy of Pediatrics, the American Association for Pediatric Ophthalmology and Strabismus, and the American Academy of Ophthalmology. Screening examination of premature infants for retinopathy of prematurity. Ophthalmology 1997;104:888-9.

11 Joint Working Party of the Royal College of Ophthalmologists and the British Association of Perinatal Medicine. Retinopathy of prematurity: guidelines for screening and treatment. Early Human Dev 1996;46:239-58.

12 Hutchinson AK, Saunders RA, O’Neil JW, et al. Timing of initial screening examinations for retinopathy of prematurity. Arch Ophthalmol 1998;116:608-12.

13 Gilbert C, Rahi J, Eckstein M, et al. Retinopathy of premaurity in middle-income countries. Lancet 1997;350:12-14.

14 Saunders RA, Bluestein EC, Sinatra RB, et al. Predictive value of posterior pole vessels in retinopathy of prematurity. 7 Pediatr Ophthalmol Strabismus 1995;32:82-5.

15 Saunders RA, Bluestein EC, Berland JE, et al. Can non-ophthalmologists screen for retinopathy of prematurity? F Pediatr Ophthalmol Strabismus 1995;32:302-4.

16 Saunders RA. A speculum for small infants. 7 Pediatr Ophthalmol Strabismus 1981;18:57-8.

17 Committee for the Classification of Retinopathy of Prematurity. An international classification of retinopathy of prematurity. Arch Ophthalmol 1984;102:1130-4.

18 Hunter DG, West CE. Last minute optics: a concise review of optics, refraction and contact lenses. Thorofare, NJ: Slack, 1996:113-14 